\title{
Perfil de susceptibilidad a los antimicrobianos en cepas de $E$ coli productoras de Shiga toxina (STEC) aisladas de infecciones humanas $y$ de alimentos
}

\author{
Marcelo Reyes S, Claudia Durán Ta , Valeria Prado J. \\ Antimicrobial susceptibility of \\ Shiga toxin producing E coli (STEC) \\ strains isolated from human \\ infections and food
}

Background: Shiga toxin-producing E coli (STEC) are zoonotic pathogens associated to sporadic episodes of bloody diarrhea, foodborne outbreaks, and Hemolytic Uremic Syndrome (HUS), with worldwide public health impact. Antibiotic use in STEC infections is controversial because of the potential to increase production and secretion of Shiga toxins. Aim: To study the in vitro antimicrobial susceptibility profile of STEC. Material and methods: The in vitro susceptibility profile against 10 antimicrobials of STEC strains isolated from 29 meat products, 20 patients with diarrhea and 9 HUS patients was studied. Minimal Inhibitory Concentrations $(\mu \mathrm{g} / \mathrm{ml})$ by agar dilution method for ampicillin, cloramphenicol, ciprofloxacin, amikacin, gentamycin, cotrimoxazol, ceftriaxone, tetracycline, fosfomycin and azithromycin were measured according to NCCLS recommendations. Results: Strains from patients with diarrhea or HUS were all susceptible to the 10 antimicrobials and only $13.7 \%$ had intermediate resistance to cloramphenicol. Strains from meat products had a similar susceptibility profile, with only $3.5 \%$ resistance to tetracycline, 3.5\% intermediate resistance to cloramphenicol and $7 \%$ to fosfomycin. All 58 strains were considered resistant to azithromycin (MIC $>32 \mathrm{ug} / \mathrm{ml}$ ). Conclusions: Similarity of susceptibility profiles between STEC strains from human and food origin suggests a role of food chain in transmission to humans (Rev Méd Chile 2004; 132: 1211-6).

(Key Words: Escherichia coli 0157; Hemolytic-uremic syndrome, Shiga toxin)

Recibido el 14 de abril, 2004. Aceptado en versión corregida el 27 de agosto, 2004.

Programa de Microbiología y Micología, Instituto de Ciencias Biomédicas, Facultad de Medicina, Universidad de Chile. Santiago de Chile.

Trabajo financiado por Proyecto Fondecyt \# 100636

aTecnólogo Médico

Correspondencia a: Dra. Valeria Prado J. Programa de Microbiología y Micología, ICBM, Facultad de Medicina, Universidad de Chile, Independencia 1027, Clasificador 7, Independencia, Santiago, Chile. Fax: 56-2-7355855. E mail: vprado@med.uchile.cl 
L a E coli productora de Shiga toxina (STEC) ha emergido como un patógeno entérico, de origen zoonótico, de considerable importancia en salud pública tanto en países desarrollados como en vías de desarrollo y cuyo principal reservorio es el ganado bovino ${ }^{1-4}$.

El rango de manifestaciones clínicas debidas a infección por STEC varía desde infección asintomática, diarrea acuosa, hasta enfermedad severa con diarrea sanguinolenta o colitis hemorrágica y síndrome hemolítico urémico (SHU) ${ }^{1,5-9}$. El SHU afecta principalmente a la población infantil, su incidencia en niños menores de 5 años en la Región Metropolitana es de 3,2 casos por $100.000^{10}$ y constituye una de las principales causas de insuficiencia renal aguda en lactantes y preescolares, con una letalidad que puede ir entre 2 y $6 \%{ }^{1-10}$.

El uso de antibióticos en las infecciones por STEC es controversial ${ }^{11}$ : se ha planteado que podrían ser un factor de riesgo de SHU ${ }^{12,13}$ y otras experiencias muestran que ciertos antimicrobianos, administrados precozmente en el curso de la infección, podrían prevenir su progresión hacia SHU 14,15 .

Un factor que influye en el desarrollo de resistencia en bacterias zoonóticas es el uso de antimicrobianos como promotores de crecimiento en el ganado ${ }^{16}$. Esta práctica produce una inevitable selección de cepas resistentes dentro de la flora comensal del tracto intestinal de los animales de crianza ${ }^{17}$ y puede tener una relevancia insospechada para la salud pública, por la posibilidad de que esta flora resistente colonice a la población humana a través de la cadena alimenticia, o mediante exposición ocupacional ${ }^{16}$.

Dentro del marco de esta controversia, nuestro interés fue aportar información actualizada respecto de la sensibilidad frente a antimicrobianos de cepas STEC, de diferente origen, que circulan en nuestro medio.

\section{MATERIALES Y MÉTODOS}

Cepas. Se estudiaron 58 cepas de STEC: 29 aisladas de muestras de alimentos, todas de serogrupos No-O157 (25 cepas de carne bovina y 4 de carne de ave) y 29 provenientes de muestras clínicas (20 cepas de pacientes con síndrome diarreico, de las cuales 5 corresponden al serogrupo 0157 y 15 al serogrupo NoO157 y 9 cepas de niños con SHU, todas serogrupo 0157) recolectadas entre los años 1998 y 2002. La identificación de STEC se inició por el diagnóstico de $\mathrm{E}$ coli siguiendo los métodos convencionales de identificación para enterobacterias ${ }^{18}$ y luego se detectó la presencia de genes codificantes de las Shiga toxinas Stx1 y Stx2 mediante técnica de RPC, siguiendo un protocolo utilizado en el Laboratorio de Microbiología y previamente descrito ${ }^{18}$.

Estudio de susceptibilidad a los antimicrobianos. Se determinó la concentración inhibitoria mínima $(\mathrm{CIM} \mu \mathrm{g} / \mathrm{ml})$, por la técnica de dilución en agar, según las recomendaciones dadas por la NCCLS 2003. Los antimicrobianos, el rango de las concentraciones y los puntos de corte utilizados en este estudio se muestran en la Tabla 1 . Una vez inoculadas las placas, fueron incubadas por 18-24 $\mathrm{h}$ a $35^{\circ} \mathrm{C}$ en atmósfera ambiente. Se utilizó como control de calidad la cepa E coli ATCC 25922 y los antimicrobianos vancomicina y eritromicina, considerando que las cepas STEC tienen resistencia natural a estos antimicrobianos.

Cálculo de CIM 50 y CIM 90. Se calculó, para cada antimicrobiano utilizado, la CIM 50 (concentración inhibitoria mínima a la cual el 50\% de las cepas detienen su crecimiento) y CIM 90 (concentración inhibitoria mínima a la cual el 90\% de las cepas detienen su crecimiento).

\section{Resultados}

La Tabla 2 muestra que las cepas de STEC aisladas de infecciones entéricas de pacientes pediátricos tuvieron elevada susceptibilidad a los antimicrobianos estudiados. Se observó una baja proporción de cepas con sensibilidad disminuida a cloranfenicol $(13,7 \%)$ y a fosfomicina $(10,3 \%)$, sin detectar cepas resistentes. Este grupo de cepas resultó $100 \%$ resistente a azitromicina. 
Las cepas de STEC aisladas de alimentos cárneos también mostraron elevada sensibilidad frente a los 10 antimicrobianos estudiados. Sólo 3,5\% mostró sensibilidad disminuida a cloranfenicol y $7 \%$ a fosfomicina. En este grupo de cepas, $3,5 \%$ resultó resistente a tetraciclina y el $100 \%$ fue resistente a azitromicina (Tabla 2).

Tabla 1. Rango de concentraciones estudiadas para cada antimicrobiano y valores de corte para evaluar sensibilidad/resistencia, según N C C LS

\begin{tabular}{|c|c|c|c|c|}
\hline \multirow{2}{*}{ Antimicrobiano } & \multicolumn{3}{|c|}{ Puntos de corte $(\mu \mathrm{g} / \mathrm{ml})$} & \multirow{2}{*}{ Rango de dilución $(\mu \mathrm{g} / \mathrm{ml})$} \\
\hline & S & I & $\mathrm{R}$ & \\
\hline Ampicilina & 8 & 16 & 32 & $0,5-64$ \\
\hline Cloranfenicol & 8 & 16 & 32 & $0,5-64$ \\
\hline Ciprofloxacina & 1 & 2 & 4 & $0,0002-8$ \\
\hline Amikacina & 16 & 32 & 64 & $0,25-128$ \\
\hline Tetraciclina & 4 & 8 & 16 & $0,25-32$ \\
\hline Gentamicina & 4 & 8 & 16 & 0,125-32 \\
\hline Cotrimoxazol & $2 / 38$ & - & $4 / 76$ & $0,03 / 0,6-8 / 152$ \\
\hline Ceftriaxona & 8 & 16 & 32 & $0,01-64$ \\
\hline Fosfomicina & 64 & 128 & 256 & $0,25-512$ \\
\hline Azitromicina & - & & - & $0,625-32$ \\
\hline
\end{tabular}

Tabla 2. Actividad in vitro de 10 antimicrobianos frente a cepas ST EC aisladas de infecciones entéricas y de alimentos

\begin{tabular}{|c|c|c|c|c|}
\hline \multirow[b]{2}{*}{ Antibiótico } & \multicolumn{2}{|c|}{$\begin{array}{l}\text { STEC infecciones clínicas } \\
n=29\end{array}$} & \multicolumn{2}{|c|}{$\begin{array}{c}\text { STEC alimentos } \\
\mathrm{n}=29\end{array}$} \\
\hline & $\begin{array}{l}\text { CIM } 50 \\
(\mu \mathrm{g} / \mathrm{ml})\end{array}$ & $\begin{array}{l}\text { CIM } 90 \\
(\mu \mathrm{g} / \mathrm{ml})\end{array}$ & $\begin{array}{l}\text { CIM } 50 \\
(\mu \mathrm{g} / \mathrm{ml})\end{array}$ & $\begin{array}{r}\text { CIM } 90 \\
(\mu \mathrm{g} / \mathrm{ml})\end{array}$ \\
\hline Cloranfenicol & 8 & 16 & 8 & 8 \\
\hline Cotrimoxazol & $0,0625 / 1,19$ & $0,0625 / 1,19$ & $0,0625 / 1,19$ & $0,125 / 2,375$ \\
\hline Ciprofloxacina & 0,0156 & 0,0312 & 0,008 & 0,008 \\
\hline Ampicilina & 2 & 2 & 2 & 4 \\
\hline Ceftriaxona & 0,312 & 0,312 & 0,0312 & 0,0625 \\
\hline Gentamicina & 1 & 2 & 1 & 2 \\
\hline Amikacina & 2 & 2 & 2 & 2 \\
\hline Tetraciclina & 4 & 4 & 2 & 4 \\
\hline Fosfomicina & 8 & 32 & 16 & 128 \\
\hline Azitromicina & 32 & 32 & 32 & 32 \\
\hline
\end{tabular}




\section{DisCUSIÓN}

El estudio de las infecciones por STEC, con fines clínicos y epidemiológicos, tiene hoy gran relevancia, sobre todo en países en vías de desarrollo, donde las condiciones de saneamiento ambiental no son óptimas, el control de calidad microbiológica de los alimentos no es riguroso y las técnicas de producción animal se están mejorando paulatinamente con miras a los mercados internacionales, donde los estándares de calidad son más exigentes.

El manejo antimicrobiano de las patologías asociadas a STEC permanece en la mayor controversia ${ }^{11}$. Estudios in vitro han demostrado que algunos tipos de antibióticos aumentarían la producción y liberación de toxinas ${ }^{12}$. Otros estudios han demostrado que la exposición a concentraciones subinhibitorias de quinolonas ${ }^{13}$ y cotrimoxazol ${ }^{11}$ producirían una gran liberación de toxinas, probablemente por activación de la respuesta SOS con sobreexpresión de genes $\mathrm{Stx}^{22}$. En el extremo opuesto, otro estudio ha descrito que se produciría una disminución de la producción de Stx al utilizar norfloxacina, fosfomicina, kanamicina, ampicilina y claritomicina en modelo de ratón murino, en donde también se demostró disminución del riesgo de complicaciones así como disminución de la excreción de STEC y de la citotoxina en deposiciones y sangre ${ }^{23}$. Desde el punto de vista clínico, se ha descrito que ciertos antimicrobianos administrados precozmente en el curso de la infección podrían prevenir la progresión hacia SHU14,15.

Debido a esta controversia, se recomienda que las infecciones por STEC no sean tratadas agresivamente con antimicrobianos, y con ello muchas cepas podrían mantener una buena susceptibilidad a diferentes antimicrobianos ${ }^{16}$.

Considerando que la transmisión de estas infecciones se relaciona con la producción animal, contaminación de carne, principalmente bovina, y el consumo de productos derivados de éstos, el patrón de susceptibilidad a los antimicrobianos puede servir como marcador epidemiológico. Otro aspecto es dar orientación para el eventual uso clínico de los antimicrobianos en pacientes con infecciones severas por STEC, como colitis hemorrágica. En estas situaciones es importante conocer la epidemiología de la resistencia antimicrobiana, para utilizar antibióticos efectivos y no agregar un factor de riesgo adicional.

Este estudio mostró que, a diferencia de otros enteropatógenos que afectan a niños chilenos como es el caso de Shigella spp, los STEC mantienen muy buena sensibilidad in vitro frente a los antimicrobianos de uso clínico, y esto se aplica tanto a las cepas de los reservorios (productos cárneos de bovino y ave) como a las de origen clínico (síndrome diarreico y síndrome hemolítico urémico). Esta similitud apoya el origen zoonótico de estas infecciones y su transmisión a través de la cadena alimentaria.

Se incluyó la evaluación de azitromicina, considerando que este azálido ha mostrado excelente actividad tanto in vitro como in vivo frente a Shigella ${ }^{19,20}$. En 1993, Gordillo y col${ }^{21}$, comunicaron una muy buena actividad de azitromicina frente a cepas de colección de patógenos entéricos, incluidas 14 cepas de STEC frente a las cuales la CIM de azitromicina fue 1 $\mu \mathrm{g} / \mathrm{ml}$. Nuestros resultados fueron diferentes, ya que registramos una CIM 90 de azitromicina de $32 \mu \mathrm{g} / \mathrm{ml}$, nivel que hemos considerado resistente, ya que si bien no se han definido los valores de corte de azitromicina para E coli, la concentración del antibiótico en la mucosa intestinal está por debajo de esa cifra ${ }^{22}$. En este sentido sería necesario estudiar a nivel molecular si existen genes de resistencia para este antimicrobiano, adquiridos horizontalmente, 0 constituye un mecanismo de resistencia natural característico de STEC, como ocurre para eritromicina y vancomicina.

La fosfomicina es utilizada en algunos países, como Japón, como tratamiento de primera elección para infecciones clínicas severas por STEC y no se ha observado emergencia de resistencia. En la literatura se ha descrito más de $90 \%$ de sensibilidad a este antimicrobiano especialmente en cepas E coli 0157:H7 y la susceptibilidad es inferior en cepas STEC de grupos No-O15723. En los STEC aislados de diferentes fuentes en nuestro medio observamos una situación similar: $90 \%$ de cepas sensibles a fosfomicina.

En las cepas que estudiamos, el $51 \%$ de las cepas de muestras clínicas eran de serogrupo 
O157 y el 49\% restante de diferentes serogrupos; ambos grupos de cepas mostraron patrones similares de susceptibilidad a los antimicrobianos.

Un estudio realizado en 1995 por nuestro grupo, mostró en cepas de STEC 21\% de resistencia a ampicilina, $20 \%$ de resistencia a cotrimoxazol, $4 \%$ de resistencia a cloranfenicol y $3 \%$ de resistencia a tetraciclina ${ }^{24}$. Podemos constatar que se han mantenido en el tiempo los niveles de resistencia a tetraciclina (3,5\%); sin embargo, ha desaparecido la resistencia a ampicilina, cotrimoxazol y cloranfenicol.

\section{REFERENCIAS}

1. López E, Prado V, O’Ryan M, Contrini M. Shigella and Shiga toxin producing Escherichia coli causing bloody diarrhea in Latin America. Inf Dis Clin of North Am 2000; 14: 41-65.

2. BESSER RE. Escherichia coli O157:H7 gastroenteritis and the hemolytic uremic syndrome: An emerging infectious disease. Annu Rev Med 1999; 50: 355-67.

3. RILEY L, REMIS S, HeLGERSON SD. Hemorrhagic colitis associated with rare Escherichia coli serotype. $\mathrm{N}$ Engl J Med 1983; 308: 681-5.

4. Karmali MA. Infection by Verocytotoxin producing Escherichia coli. Clin Microbiol Rev 1989; 2: 15-38.

5. Griffin PM, TAUXe RV. The epidemiology of infections caused by Escherichia coli 0157:H7, other enterohemorrhagic E coli, and the associated hemolytic uremic syndrome. Epidemiol Rev 1991; 13: 60-98.

6. Siegler RL. The hemolytic uremic syndrome. Ped Nephrol 1995; 42: 1505-29.

7. Nataro JP, Kaper JB. Diarrheagenic Escherichia coli. Clin Microbiol Rev 1998; 11: 142-201.

8. López EL, Devoto S, Fayad A, Canepa C, Morrow AL, Cleary TG. Association between severity of gastrointestinal prodrome and long term prognosis in classic hemolytic uremic syndrome. J Pediatr 1992; 120: 210-5.

9. Pickering L, Obrig T, Stapleton B. Hemolyticuremic syndrome and enterohemorrhagic Escherichia coli. J Pediat Infect Dis 1994; 13: 459-76.
Este estudio muestra que las cepas STEC, tanto de origen animal como asociadas a infecciones clínicas, circulantes en nuestro medio presentan una elevada susceptibilidad a los antimicrobianos y que en el caso de cepas STEC de infecciones gastrointestinales el perfil ha mejorado en relación a observaciones anteriores. Esto podría estar reflejando el manejo más cuidadoso de antibióticos en este tipo de patología, con abstención del uso de antimicrobianos en casos innecesarios, así como también un mejoramiento técnico del proceso de producción animal.

10. Vizcaya MC, Sandoval C, Salin MP, Prado V. Impacto del síndrome hemolítico urémico en las distintas áreas de salud de la región metropolitana. Rev Chil Infect 1996; 13: 223-30.

11. Safdar N, Said A, Gangnog R, Maki D. Risk of hemolytic uremic syndrome after antibiotics treatment of Escherichia coli 0157:H7 enteritis. JAMA 2002; 288: 996-1001.

12. Walterspeil J, Ashrenazi S, Morrow A, Cieary tG. Effect of subinhibitory concentrations of antibiotics on extracellular Shiga like toxin 1. Infection 1992; 20: 25-9.

13. Patrick TK, Colin RH, Barer M. Induction of type 2 Shiga toxin synthesis in Escherichia coli 0157 by 4-quinolones. Lancet 1999; 353: 1588-99.

14. IKeda $K$, Ida $O$, Kimoto $K$, Takatoriage $T$, NaKanishi $\mathrm{N}$, TATARA K. Effect of early fosfomycin treatment on prevention of hemolytic uremic syndrome accompanying Escherichia coli 0157:H7 infection. Clin Nephrol 1999; 52: 357-62.

15. Shiom M, Togawa M, Fujta K, Murata R. Effect of early oral fluoroquinolones in hemorrhagic colitis due to Escherichia coli 0157:H7. Peditr Int 1999; 41: 228-32.

16. SCHROEDER CM, ZHAO C. Antimicrobial resistance of Escherichia coli $\mathrm{O} 157$ isolated from human, cattle, swine and food. App Environ Microbiol 2002; 68: 576-81.

17. Van Den Bogaard AE, Stobbering EE. Antibiotic usage in animals: impact on bacterial resistance and public health. Drugs 1999; 58: 589-607.

18. Vidal M, Carreño M, Vidal R, Areliano C, Solari V, Prado V. Evaluación de técnicas moleculares 
e inmunoenzimáticas para la detección de E coli enterohemorrágica en brotes de toxi-infecciones alimentarias. Rev Méd Chile 2002; 130: 603-9.

19. Basuaddo W, Arbo A. Randomized comparison of azithromycin versus cefixime for treatment of shigellosis in children. Pediatr Infect Dis J 2003; 22: 374-7.

20. Khan WA, Seas C, Dhar U, Salam MA, Bennish ML. Treatment of shigellosis: V. Comparison of azithromycin and ciprofloxacin: a double-blind, randomized, controlled trial. Ann Intern Med 1997; 126: 697-703.

21. GordiLo M, Singh K, MurRia B. In vitro activity of azithromycin against bacterial enteric patho- gens. Antimicrob Agents Chemother 1993; 37: 1203-5.

22. Foulds G, Shepard RM, Auen RH, Ferraina RA. Transintestinal elimination of azithromycin in dogs (Abstract 220). In: Proceedings of the Fifth European Congress of Clinical Microbiology and Infectious Diseases, Oslo, 1991.

23. Tsuboi I, Hirohisa I. Antibiotic susceptibility of enterohemorrhagic Escherichia coli 0157:H7 isolated from an outbreak in Japan in 1996. Antimicrob Agents Chemother 1998; 42: 431-2.

24. Prado V, Basualdo W, arellano C, Martínez D, Levine MM. Susceptibilidad in vitro de Escherichia coli enterohemorrágica frente a 11 antimicrobianos. Rev Méd Chile 1995; 123: 1085-90. 